\title{
Twin ostial openings in the left posterior aortic sinus: a pictorial overview of coronary revascularisation and aortic valve replacement in a patient with absent left main artery
}

\author{
Nasir Hussain, ${ }^{1,2,3}$ Atiq Rehman, ${ }^{3,4}$ Faisal H Cheema $^{5}$
}

${ }^{1}$ Hartford Hospital, University of Connecticut, Hartford, Connecticut, USA ${ }^{2}$ Department of Internal Medicine, Saint Joseph Hospital, Chicago, Illinois, USA ${ }^{3}$ Department of Cardiothoracic Surgery, College of Physicians and Surgeons of Columbia University, New York Presbyterian Hospital, New York, New York, USA ${ }^{4}$ Sarasota Memorial Hospital, Sarasota, Florida, USA ${ }^{5}$ Department of Cardiothoracic Surgery, University of Maryland Medical Centre, Baltimore, Maryland, USA

\section{Correspondence to} Dr Nasir Hussain, connect2nasir@gmail.com

Accepted 1 March 2014

\section{DESCRIPTION}

Congenital absence of the left main (LM) with left anterior descending (LAD) and left circumflex (LCX) arteries arising directly from the left sinus of Valsalva with their separate ostia is an extremely rare anomaly with an estimated incidence rate of around $0.4 \%$ of the cardiac catheterisations performed. ${ }^{1}$ A 73-year-old man presented with a 8 -month history of exertional dyspnoea. Preoperative diagnostic workup demonstrated severe aortic stenosis on transthoracic echocardiography, while the coronary catheterisation depicted separate ostia with critical ostial stenosis for the LAD and LCX arteries (figure 1A-D). The patient underwent coronary artery bypass graft with left internal mammary artery anastomosis to the LAD and a reverse saphenous vein graft anastomosis to the obtuse marginal branch of LCX. A concomitant aortic valve replacement (AVR) was also performed and separate ostia were clearly identified and preserved while seating the new prosthesis (figure 2A, B). Thus, the presentation of an absent LM artery may affect the clinical management, especially in cases of AVR. Injection of the contrast dye into the left posterior aortic sinus during the caudal left anterior oblique or lateral projections of the coronary angiography helps in making a diagnosis for this anomaly. ${ }^{1}$ Operator should ensure that selective decannulation and catheter-related vasospasm, by careful monitoring of pressure tracing during cardiac catheterisation, is differentiated from true absence of LM. ${ }^{2}$
A

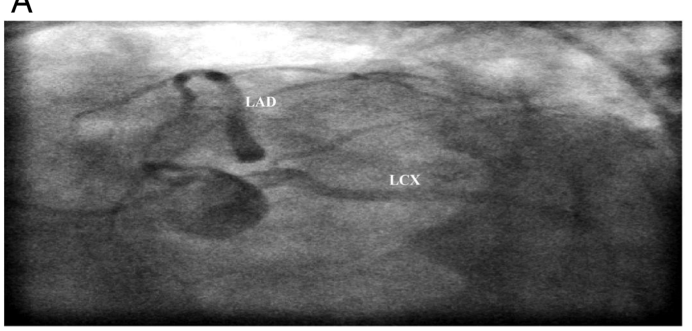

C

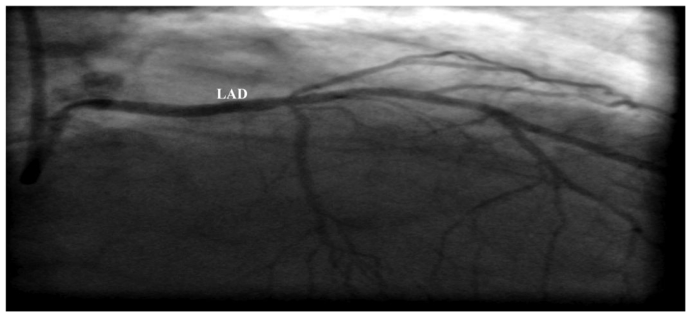

B

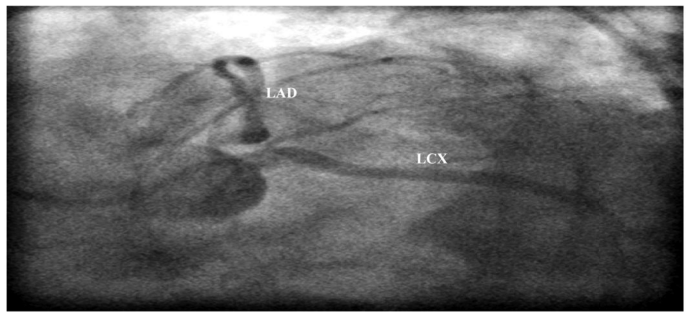

D

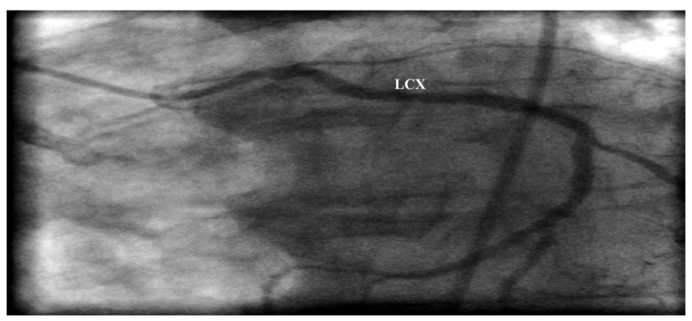

Figure 1 Images from the cardiac catheterisation laboratory. (A and B) Images taken in left anterior oblique (LAO) caudal view and depict separate ostial origins with critical ostial stenosis for left anterior descending (LAD) and left circumflex (LCX). (C and D) Images taken in LAO cranial view and demonstrate absent left main. 

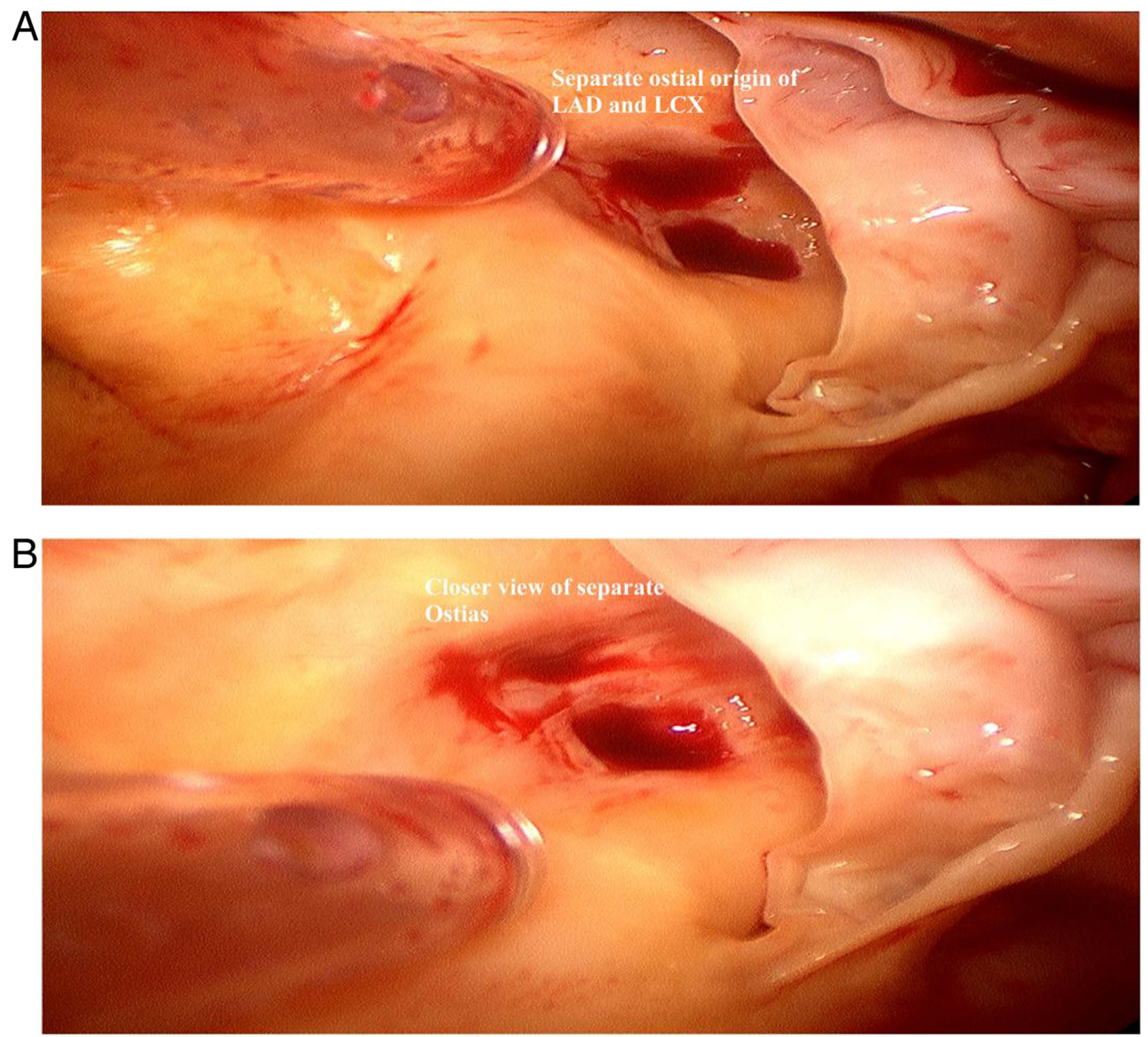

Figure 2 Images from the operating room. (A and B) Intraoperative images that depict separate ostial origins of the left anterior descending (LAD) and left circumflex (LCX) from the left sinus of Valsalva.

\section{Learning points}

- It is particularly important for the physicians to be aware about the true congenital absence of the left main and be able to differentiate the origins of separate ostia for left anterior descending and left circumflex on coronary angiograms.

- Clear identification of the ostia during an aortic valve replacement (AVR) is essential since the anomalous origin of the separate ostia could be from the same or different sinuses of Valsalva; therefore, extra caution should be taken in seating the prosthetic valve during AVR lest the struts or the sewing ring of the valve inadvertently compromises one of the ostia.

- In addition, separate ostial identification is also important in cases of AVR for aortic insufficiency for direct ostial infusion of cardioplegia (if a retrograde coronary sinus cardioplegia catheter is not used).
Contributors $\mathrm{NH}$ and $\mathrm{FHC}$ were involved in writing and preparation of the manuscript. AR contributed to preparation of the manuscript and was directly involved in the patient care and performed the surgery on the patient.

\section{Competing interests None.}

Patient consent Obtained.

Provenance and peer review Not commissioned; externally peer reviewed.

\section{REFERENCES}

1 Topaz O, DiSciasscio G, Cowley MH, et al. Absent left main coronary artery: angiographic findings in 83 patients with separate ostia of the left anterior descending and circumflex arteries at the left aortic sinus. Am Heart $J$ 1991;122:447-52.

2 Longenecker CG, Reemtsma K, Creech $0 \mathrm{Jr}$. Surgical implication of single coronary artery: a review and two case reports. Am Heart J 1961;61:383-6. 